\title{
Intramammary propolis formulation for subclinical mastitis prevention and treatment in dairy cows
}

\section{Introduction}

Bovine mastitis is the main cause of economic losses in dairy industry and a food safety concerns due to a wide spread antimicrobials use. Although there are many treatment options available, there is a lack of effective treatment concerning the subclinical mastitis in dairy cows. Mastitis prevalence in dairy herds has resulted in the extensive use of antibiotics. The greatest risk lays in development of antibiotic resistant bacteria, but it also presents an obstacle for organic milk production. Many subclinical mastitis treatment studies ${ }^{1,2}$ proposed alternatives to conventional antimicrobial treatment solutions. Propolis is a resinous mixture that honeybees collect from trees and various plants. It is interesting due to its numerous biomedical effects; immune modulating, antioxidative and antimicrobial. Main goals of our work are development and research of the innovative concept of intramammary nonalcoholic propolis formulation as alternative to existing antimicrobial prevention and treatment of subclinical mastitis. The development of formulation for dairy cows was conducted together with field veterinarians and dairy farmers. Our research consists of four main frame works.

\section{Propolis formulation development}

Raw propolis has been collected from ecologically clean parts of Croatia then pulverized to micro particles. Extraction was done with polyethylene glycol solution and then filtered. Different concentrations of propolis $(0.1,0.5,1,3,5$ and $10 \%)$ have been prepared by an innovative method protected by "Hedera" Ltd.

\section{Analysis of poly phenol content in propolis formula- tion}

Extracts of native propolis were analyzed by Shimadzu GC-MS Ultra Gas Chromatograph Mass Spectrometer to quantify chemical compounds responsible for differences in antioxidative capacity. The quantification was carried out by area normalization with internal standards for each one of 7 different compounds analyzed. Results are presented as \% total ion current (TIC) for each compound. Extracts differed in quantity of different compounds responsible for antioxidative properties and biological activity.

\section{In vitro study of antimicrobial efficacy against common mastitis pathogens}

Tests show good in vitro activity of nonalcoholic propolis formulation against selected gram-positive bacteria (Staphylococcus aureus, coagulase-negative staphylococci), with minimum inhibitory concentrations (MICs) of propolis in the range from 16 to $64 \mathrm{mg} / \mathrm{L}$ $(0,002-0,007 \mathrm{oz} / \mathrm{gal})$. Significantly lower activity was determined for E. Coli and Pseudomonas aeruginosa with MICs higher than $5000 \mathrm{mg} / \mathrm{L}(0,608 \mathrm{oz} / \mathrm{gal})$.

\section{Clinical trial on holstein cows}

Five dairy herds ( 800 quarter milk samples per farm) were analyzed for microbiology, somatic cell count and chemical composition. Propolis solution was homogenous, viscous and stabile. Initially we started the clinical tests with $10 \%$ propolis formulation but due to prominent udder tissue changes (redness, swelling, increased
Volume 3 Issue 5 - 2016

\author{
Goran Bacic,' Nino Macesic,' Lada Radin,' \\ Jasna Aladrovic,' Kresimir Matanovic,' \\ Tomislav Masek,' Diana Brozic,' Miroslav \\ Benic, ${ }^{2}$ Bozo Radic, ${ }^{3}$ Iva Bacic, 'Jelena Suran ${ }^{3}$ \\ 'Faculty of Veterinary Medicine, Croatia \\ ${ }^{2}$ Croatian Veterinary Institute, Croatia \\ ${ }^{3}$ Hedera Ltd, Croatia
}

Correspondence: Goran Bacic, Faculty of Veterinary Medicine, Veterinary College Zagreb University, Croatia, Email buch800@yahoo.com

Received: July 04, 2016 | Published: July 18, 2016

temperature) we switched to $1 \%$ formulation. Incidence of subclinical mastitis as well as milk quality and oxidative stress parameters were monitored through on farm quarter milk sampling. Our target population on each farm was high somatic cell count (SCC)/infected cow group. We treated randomly selected cows from "problem" group with $1 \%$ solution during three $12 \mathrm{hrs}$ period (during morning, evening and again morning milking). Milk from each quarter was sampled for microbiology, SCC and oxidative stress parameters prior to each treatment . The most common intramammary infection pathogens before treatment were Corynebacterium spp.(33.3\%), Streptococcus uberis (27.8\%), Streptococcus spp.(22.2\%), Pasteurella spp.(11.1\%) and pathogenic yeasts $(5.5 \%)$. Prevalence of intramammary infection before first propolis application was $28.6 \%, 15.9 \%$ before second application and $4.8 \%$ before the third. Seven days after first application control sampling was performed and infection.

\section{Conclusion}

So far $1 \%$ intramammary propolis formulation showed high antibacterial and antioxidant activity in vitro and in vivo. It may be good alternative to conventional antimicrobials used for subclinical mastitis prevention and treatment in dairy cows. More final results will be published soon.

\section{Acknowledgements}

None.

\section{Conflict of interest}

Author declares that there is no conflict of interest.

\section{References}

1. Diarra MS, Block G, Rempel $\mathrm{H}$, et al. In vitro and in vivo antibacterial activities of cranberry press cake extracts alone or in combination with $\beta$-lactams against Staphylococcus aureus. BMC Complement Altern Med. 2013;13:90.

2. Vitor da Rocha Sperotto, Anelise Levay Murari, Décio Adair Rebellatto da Silva, et al. Activity of Decoction of Achyrocline satureioides DC (Lam.-Asteraceae ("macela") against Standard and Isolated Bacteria from Bovine Mastitis. Acta Sci Vet. 2012;40(3):1052. 